\title{
Properties of Mira Photospheres
}

\author{
M.Scholz \\ Institut für Theoretische Astrophysik, Universität Heidelberg, Germany
}

The first crude pictures of the structure of Mira photospheres were derived from velocity analyses of emission and absorption lines appearing shifted, doubled and doppler-broadened in Mira spectra. Positions and shapes of profiles change with phase and often do not repeat in successive cycles. Non-repeating cycles are also found in colors (e.g., TiO bands in Fig. 9 of Spinrad \& Wing 1969) and in light curves and present, of course, a problem in matching specific observed and modeled cycles. The large geometric extension of Mira photospheres was demonstrated by monochromatic radius observations of $o$ Ceti and R Leo by Labeyrie et al. (1977) and Bonneau et al. (1982) which, however, did not systematically follow the stars through different phases and cycles.

The structure of a Mira photosphere is essentially determined by the propagation of shock fronts. A shock front enters the bottom of the photosphere once each cycle and travels outwards. The stellar material is accelerated by the shock wave and falls back after some time, and it is heated by dissipation of shock energy. As a result, the structure of the Mira photosphere differs substantially from that of a non-variable star. (i) There are density discontinuities at the shock positions and very flat density gradients in between, yielding a geometrically very extended configuration. (ii) The photosphere has a pronounced outflow/infall velocity stratification. (iii) There is a thin hot layer just behind the shock front producing typical emission lines plus, depending on how slow or fast relaxation towards equilibrium occurs, a more or less extended non-equilibrium region possibly affecting the absorption line spectrum. See Fig. 1 of Scholz (1992) for a typical stratification where instantaneous relaxation to equilibrium is assumed because of still quite insufficient knowledge of relaxation processes (cf. Beach et al. 1988; Bessell \& Scholz 1989).

The predictions presented here of the behavior of typical observable features are based on unpublished self-excited pulsation models of P.R. Wood (cf. Wood 1974, 1990) the photospheric portion of which is a non-grey, spherically extended configuration in local thermodynamic and radiative equilibrium (cf. Scholz 1992) not including the emission-line forming zones. Luminosities $L$, effective temperature $T_{\text {eff }}$ and radii of selected photospheric layers of a typical model sequence covering two successive cycles, with parameters close to $o$ Ceti $\left(1 M_{\odot}\right.$, fundamental mode pulsation, $\mathrm{M}$ type composition), are shown in Fig. 1. Here, the stellar radius $R$ is the Rosseland $\bar{\tau}=1$ radius which is practically identical with the observable continuum radius. Fig. 1 demonstrates the slow and regular pulsation of the star in terms of $R$ whereas the shock fronts move outward in a fairly erratic manner and do not repeat in successive cycles. The positions of the photospheric surface (i.e., the layers below an outer wind envelope, say around $\bar{\tau}=10^{-5}$ ) and of monochromatic $\tau_{\lambda}=1$ radii at strong $\mathrm{TiO}$ ab- 


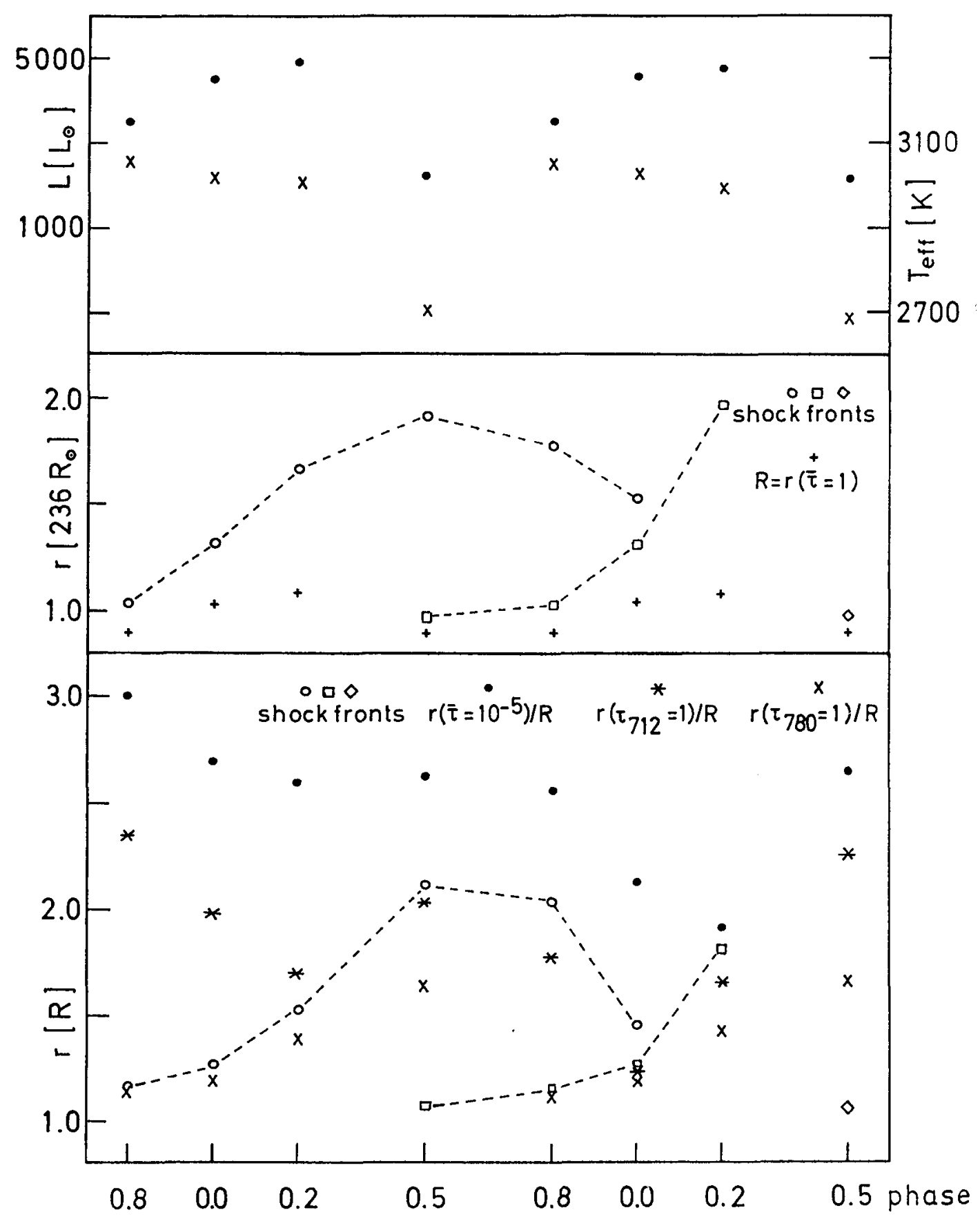

Fig. 1. Luminosities $L$ (dots), effective temperatures $T_{\text {eff }}$ (crosses) and radii of selected photospheric layers (symbols given in the panels) of amodel sequence covering two successive cycles (see text). 
sorption (e.g., $712 \mathrm{~nm}$ ) are obviously dominated by the details of shock propagation. In contrast, the positions of monochromatic radii at moderate $\mathrm{TiO}$ absorption (e.g., $780 \mathrm{~nm}$ ) vary more slowly and more regularly. As one considers colors measuring the same $\mathrm{TiO}$ features, one finds a behavior similar to that given in Fig. 9 of Spinrad \& Wing (1969) with a tight correlation with monochromatic radii: the farther above the continuum the feature is formed the stronger the band appears on the details of shock propagation. (Note that the monochromatic radii and colors calculated here should be considered with caution as they are based on frequency-averaged absorption coefficients and as they do not take into account outflow/infall velocity broadening of lines).

The recent model study of Scholz (1992) and high-resolution observations of Mira spectra (see Scholz 1992 for a list of references) have also shown that the profiles of absorption lines of different strengths from selected atoms and molecules react sensitively to the variations of the outflow/infall velocity field from phase to phase and from cycle to cycle. Thus, it is obvious that observations of monochromatic radii and colors probing absorption features of different strengths (including the position and slope of the continuum), covering the phases of successive cycles, provide accurate informaiton about the details of the structure of a Mira photosphere, in particular when these observations are supplemented by simultaneous measurements of absorption-line profiles and of emission-line velocity shifts.

\section{References:}

Beach, T.E., Willson, L.A. 1988, ApJ, 329, 241.

Bessell, M.S. \& Scholz, M. 1989, in Evolution of Peculiar Red Giant Stars (IAU Colloq. 106), eds. H.R.Johnson \& B.Zuckerman, Cambridge University Press, Cambridge, p.67

Bonneau, D. Foy, R., Blazit, A. \& Labeyrie, A. 1982, A\&A, 106, 235

Labeyrie, A., Koechlin, L., Bonneau, D., Blazit, A. \& Foy, R. 1977, ApJ, 218, L75.

Scholz, M. 1992, A\&A, 253, 203

Spinrad, H. \& Wing, R.F. 1969, ARA\&A, 7, 249

Wood, P.R. 1974, ApJ, 190, 609

Wood, P.R. 1990, in From Miras to Planetary Nebulae, eds. M.O. Mennessier \& A. Omont, Frontieres, Gif, p.67 